\title{
Trabajo, territorio y cuerpos en clave \\ neodesarrollista. Argentina, 2002-2016
}

\author{
Mariano Féliz,* Juliana Agustina Díaz Lozano**
}

Perfiles Latinoamericanos, 26(52) | 2018

DOI: $10.18504 / \mathrm{pl} 2652-005-2018$

Recibido: 28 de septiembre de 2016

Aceptado: 26 de septiembre de 2017

\section{Resumen}

Desde 2002, Argentina consolidó un proyecto neodesarrollista basado en el saqueo de la naturaleza, la superexplotación del trabajo, la reconfiguración de la reproducción social, y la refuncionalización del lugar de las mujeres, a partir de nuevas modalidades en el uso de la fuerza laboral femenina asalariada y no asalariada. Esta investigación aborda dicho neodesarrollo, pero desde una perspectiva que articula la coconstitución del capitalismo, el patriarcado y el saqueo de riquezas naturales en la periferia contemporánea. Para esto el análisis enlaza el marxismo latinoamericano con los debates feministas en cuanto al lugar central e invisibilizado del trabajo de reproducción y de cuidados.

\begin{abstract}
Since 2002, Argentina consolidated a neodevelopmentalist project, which is based in the plundering of nature, superexplotation of labor and the reconfiguration of social reproduction. This article analyzes neodevelopmentalism in Argentina from a perspective that systematically articulates the co-constitution of capitalism, patriarchy and plundering of natural riches in the contemporary periphery. We'll follow up on this study through an analytical perspective that articulates Latin American Marxism with feminist debates on the key but unseen work of reproduction or care.
\end{abstract}

Palabras clave: neodesarrollismo, recursos naturales-explotación, trabajo de reproducción, políticas públicas, patriarcado, capitalismo, Argentina.

Keywords: Neodevelopmentalism, natural resources-exploitation, reproductive labour, public policies, patriarchy, capitalism, Argentina.

* Doctor en Ciencias Económicas (Universidad de París xiI/Nord) y doctor en Ciencias Sociales (Universidad de Buenos Aires). Profesor Ordinario por concurso, Facultad de Humanidades y Ciencias de la Educación, Universidad Nacional de La Plata (UNLP) e Investigador Independiente en el Centro de Investigaciones Geográficas del Instituto de Investigaciones en Humanidades y Ciencias Sociales (cIGIdiHCS) del CONICET y la UNLP | marianfeliz@gmail.com

** Licenciada y Profesora de Periodismo y Comunicación Social por la Universidad Nacional de La Plata. Becaria doctoral del coniceT en el Instituto Interdisciplinario de Estudios de Género, Facultad de Filosofía y Letras, Universidad de Buenos Aires (UBA) | diazlozano.juliana@gmail.com 


\section{Introducción}

\section{A}

mérica Latina y el Caribe han sido atravesados por procesos sociopolíticos neodesarrollistas con grados diversos de radicalidad y quiebre respecto del neoliberalismo y los niveles de participación popular (Féliz, 2015a). Son procesos que han sido muy debatidos y analizados, pero raramente problematizados desde una perspectiva que articule, de manera sistemática, la coconstitución del capitalismo y el patriarcado como elementos integrales en la conformación de las estrategias neodesarrollistas y como clave para comprender a estas en sus potencialidades y límites. Tampoco hay estudios que se propongan desentrañar integralmente la articulación entre las lógicas de construcción del neodesarrollo y las modalidades de saqueo de las riquezas naturales y los bienes comunes, de la superexplotación de la fuerza de trabajo y de la explotación del cuerpoterritorio de las mujeres y cuerpos feminizados.

Este artículo avanza en tal tipo de abordaje pues analiza cómo la construcción del proyecto neodesarrollista en Argentina (como parte del proceso regional) integró esas facetas de la reproducción social del capitalismo periférico en la era de la transnacionalización del capital.

El trabajo se organiza del siguiente modo. Primero se discute el debate histórico sobre la articulación del capitalismo y su "etapa" imperialista, con las modalidades de apropiación del trabajo. Luego se revisa la lectura del desarrollo en la periferia, en especial en América Latina, incorporando los debates sobre la articulación entre los llamados trabajos productivo y reproductivo o de cuidados. Posteriormente se destaca la preeminencia del saqueo como forma general de la apropiación del trabajo y de la naturaleza en la actualidad, para luego describir la modalidad específica de tales articulaciones en la construcción hegemónica del proyecto neodesarrollista en Argentina a partir de 2002. La cuarta sección se focaliza en el papel del Estado como engrane de la opresión y explotación de las mujeres en la constitución misma del neodesarrollo, en especial mediante las políticas sociales y laborales. Enseguida se discute la modalidad en la que el saqueo y la apropiación violenta del cuerpo-territorio de las mujeres son orgánicamente constitutivos del proyecto neodesarrollista argentino. Por último, se presentan unas breves conclusiones.

\section{Capitalismo, imperialismo y la apropiación del trabajo y de los trabajos}

La historia del capitalismo ha sido la del saqueo de los bienes comunes y de la apropiación de los cuerpos —en especial, el de las mujeres (Federici, 2011)_ 
para consolidar la valorización del capital. Marx presentó la acumulación originaria del capital como el fundamento de esa historia de apropiación violenta. La acumulación primitiva, analizada desde lecturas marxistas y feministas como la de Federici (2011), es definida como la concentración de trabajadores y trabajadoras explotables y de capital, pero también como la acumulación de diferencias y divisiones dentro de la clase trabajadora. En este proceso de múltiples separaciones, las jerarquías de género, raza y edad pasaron a ser constitutivas de la dominación de clase y de la formación del proletariado moderno.

A comienzos del siglo xx, Rosa Luxemburgo llevó al debate el papel del imperialismo en la reproducción ampliada de las relaciones capitalistas. Con ello enfatizaba el papel fundamental que tendría en este proceso la ocupación de espacios sociales no capitalistas como un camino para expandir las posibilidades de valorización del valor. Así, la acumulación originaria sería entonces un proceso recurrente — presente más allá de una primera etapa del capital — en el que se renovarían de continuo (De Angelis, 2012) la acumulación originaria del capital, la apropiación del cuerpo-territorio de las mujeres (Segato, 2014; Gargallo, 2002; Hernández, 2014) y la división sexual del trabajo, como condiciones para la reproducción ampliada del capital (Federici, 2013). En las últimas décadas, a partir de una revisión y reescritura de la historia colonial en clave feminista, se ha comenzado también a señalar el papel de la conquista en la constitución de formas específicas de patriarcado en los territorios conquistados, lo que produce diversos modos de colonialidad del género que aún persisten (Lugones, 2008; Segato, 2011).

Las lecturas marxistas clásicas ignoraron el papel que en la historia del capitalismo tiene la apropiación del cuerpo y el trabajo de las mujeres a fin de garantizar condiciones básicas para la ampliación infinita del valor. En cambio, los estudios feministas en toda su diversidad y debates (Amorós, 1985; Hartmann, 1985; Haraway, 1995; Millet, 2010; Izquierdo, 1983; Federici, 2011; entre tantas otras) sí han discutido esa condición de posibilidad para la constitución del capitalismo, destacando cómo el patriarcado se constituyó - y se constituye todos los días - en una relación inseparable con el capitalismo $(\mathrm{Ru}-$ bin, 1975; Chaneton, 2007).

Desde esta perspectiva, el patriarcado actual se define como un sistema que se asienta en la explotación del trabajo de las mujeres y la dominación económica, y en la explotación sexual, la heterosexualidad obligatoria y la pérdida de libertades y posibilidades de decisión sobre la vida propia. La dominación masculina se ejerce sobre las mujeres y todo sujeto feminizado que no cumpla con el estereotipo de varón, burgués, occidental, blanco, adulto, heterosexual y propietario (Haraway, 2004; Lorde, 2003). En este proceso, la invisibilización 
del trabajo reproductivo y la carga de este casi exclusivamente en el cuerpo de las mujeres es medular para el sostén y reproducción del sistema.

En efecto, el llamado trabajo reproductivo — de la fuerza de trabajo, de la vida familiar (Pérez Orozco, 2014) y, más ampliamente, de producción comunitaria, o de lo "común", en palabras de Gutiérrez (2015) — funge como sostén invisible del trabajo productivo de plusvalía. Crea las bases sociales de la producción de esta última y contribuye a la producción y reproducción de la mercancía más valiosa para el capital: la fuerza de trabajo. En su formación histórica, el capitalismo se configuró en vinculación inseparable con las relaciones patriarcales colocando a las mujeres como garantes de la producción de las condiciones básicas para la reproducción de la fuerza de trabajo que - como capital variable - crea periódicamente valor económico excedente, es decir, plusvalor. Se conformó así una cadena de explotación que va del capital al trabajo, y de varones a mujeres, que crea otras tantas formas de la reproducción del capital. Al decir de Flora Tristán, "La mujer es la proletaria del proletariado [...] hasta el más oprimido de los hombres quiere oprimir a otro ser: su mujer" (Tristán, 1993). Con el tiempo se ha consolidado una coconstitución orgánica entre capitalismo y patriarcado - y extractivismo/racismo/colonialidad-, construyendo un sistema social de múltiples opresiones (Valdés, 2001) y formas históricas nuevas.

Precisamente, a lo largo de la historia de la relación del capital, que se materializa en la formación social capitalista, la relación contradictoria entre capitaltrabajo y entre varones-mujeres, se ha estructurado en modalidades diversas. Esto ha sido posible por el carácter meta-estable del patriarcado (Amorós, 1985), en tanto sistema capaz de adaptarse a distintos contextos económicos y sociales que incorpora demandas para asegurar así su reproducción. Esas articulaciones cambiaron también por las luchas sociales contra el imperio del capital y tuvieron expresiones a través del Estado y los proyectos sociales hegemónicos. En este sentido, los movimientos de mujeres y feministas se han esforzado por denunciar la institucionalización y cooptación simbólica por parte del Estado y organismos internacionales de las demandas para que se reconozcan la opresión y dominación en clave de géneros (Fraser, 1997). Admitir este proceso no invalida las transformaciones en la organización social y económica que la movilización popular y el activismo del movimiento de mujeres han impulsado. Estas últimas consiguieron más bien visibilizar las complejidades de las/los/les sujetos, el modo en que las relaciones de clase, géneros y raza se entrecruzan generando opresiones imbricadas, en particular en América Latina y el Caribe (ALyC), para las mujeres pobres, indígenas, afrodescendientes, mestizas, lesbianas, migrantes. Por ello, desde los feminismos de color y decoloniales se aportó la perspectiva de intersección o imbricación de las relaciones de clase, género 
y raza, que se produce de una manera específica y situada en cada formación histórica, y que posibilita visualizar la complejidad y consustancialidad de las relaciones sociales y de los cómo es que los/las/les sujetos las experimentan y/o resisten (Viveros, 2016).

\section{Imperialismo, desarrollismo y patriarcado en la periferia}

Los procesos de neodesarrollo recientes en la región corresponden a las estrategias de desarrollo capitalistas contemporáneas impulsadas por las fracciones hegemónicas en las clases dominantes capitalistas. Tales estrategias han variado a lo largo de la historia, lo que da cuenta de los cambios en las correlaciones de fuerzas, en los modos de la acumulación de capital a escala internacional y en las modalidades de la explotación del trabajo, del cuerpo-territorio de las mujeres y de la naturaleza. Para comprender la actual estrategia dominante, es necesario resumir sus transformaciones en el pasado reciente.

Desde comienzos del siglo xx, el proyecto del capital imperialista tuvo como sucesoras las variantes del capitalismo fordista-keynesiano, que en Alyc han llevado una impronta desarrollista (Féliz, 2015a). En esta etapa se pretendió consolidar una división sexual del trabajo en el que las mujeres eran el fundamento del trabajo reproductivo en los hogares donde el único proveedor de ingresos era un varón adulto, en general, el esposo. Esta división del trabajo entre el espacio público — del varón, heterosexual, blanco, asalariado, remunerado - y el espacio privado - de la mujer, heterosexual, blanca, no asalariada, no remunerada - se fortaleció por el desarrollo de una política laboral y social que canalizaba los derechos sociales del Estado de bienestar a través del trabajador asalariado (Dalla Costa, 2009), la cual se combinaba con la implementación de políticas culturales y educativas que fundamentaban tal división sexual del trabajo en condiciones biológicas y supuestas aptitudes naturales (Millet, 2010). Una división también atravesada por la colonialidad y el racismo, en particular en ALyc, donde las fracciones migrantes y originarias de los pueblos de la región enfrentaron históricamente el acrecentamiento de estas modalidades de separación.

Hacia fines de los años sesenta, la avanzada neoliberal pretendió la reorganización general del capital en una suerte de superación dialéctica de las contradicciones que le son inmanentes. La crisis del proyecto keynesiano/desarrollista abrió el espacio para transformaciones potencialmente radicales (no necesariamente progresivas) en la estructura social (Dalla Costa, 2009; Cleaver, 1992; Harvey, 2005). El deterioro de las condiciones del empleo asalariado en Argentina (Féliz \& Pérez, 2007), a su vez, fortaleció el ingreso de las mujeres al 
mercado laboral mientras que les aumentaba la carga del trabajo reproductivo, en la medida en que el Estado abandonaba o reducía su papel como integrador o proveedor de beneficios (Anzorena, 2013a; Rodríguez Enríquez, 2011). Esto fue particularmente cierto en Argentina donde el Estado de bienestar periférico alcanzó mayores niveles de desarrollo y había suplantado o complementado a las familias — sobre todo las urbanas - en algunos trabajos de reproducción. La crisis del Estado de bienestar en este país trasladó de nuevo a los hogares, y en especial a las mujeres, las tareas de reproducción que habían sido parcialmente estatizadas.

Ahora bien. Desde el movimiento de mujeres se había criticado el modelo de familia fordista, que se sostenía en la división sexual del trabajo y de las esferas pública y privada, en el salario familiar, o salario indirecto, y las políticas laborales de la posguerra (Dalla Costa \& James, 1972). Con el neoliberalismo este debate adquirió otros matices. Desde lecturas feministas hoy se advierte cómo las críticas al salario familiar han aportado para legitimar las reformas neoliberales y el capitalismo flexible, del cual el trabajo precario asalariado de las mujeres es una de sus bases (Fraser, 1997). Estas miradas señalan que la salida obligada al mercado laboral, lejos de liberar a las mujeres, ha resultado en dobles y triples jornadas, $y$ ha incrementado brutalmente su carga de trabajo cotidiano. En ALyc este proceso se caracteriza por la superexplotación del trabajo remunerado en las economías dependientes (Marini, 1973), algo que se multiplica cuando se trata del trabajo remunerado y no remunerado de las mujeres, sobre todo si son migrantes.

La mundialización neoliberal capitalista acrecentó el ingreso de las mujeres a los mercados de empleo en todo el globo, en general destruyendo sus anteriores modos de existencia mediante la recreación de formas de acumulación originaria (Cielo \& Vega, 2015; Falquet, 2016b) o acumulación por desposesión (Harvey, 2005). Esto significó la multiplicación de la circulación internacional de las mujeres pobres, las cuales toman a su cargo gran parte del trabajo reproductivo en los países de destino. Desde los estudios feministas, este proceso ha recibido distintos nombres: hetero-circulación de las mujeres (Falquet, 2016a), movilización nacional e internacional de mujeres de servicios (Tabet, 2005), o externalización de tareas reproductivas (Sassen, 1991). Como niñeras, criadas o auxiliares de salud, estas mujeres se volvieron cada vez más esenciales en lo que constituye una verdadera internacionalización de la reproducción social (Falquet, 2016a) en tareas abandonadas por el Estado, o realizando el trabajo doméstico para mujeres insertas en el mercado laboral. Esto constituye una porción de los modos de reproducción a escala ampliada de la apropiación del cuerpo de las mujeres por parte de los varones y el capital (Segato, 2014), lo que incluye la prostitución y trata laboral. 
Las transformaciones globales en el capitalismo a través del proyecto neoliberal forjaron novedosas formas de producción, apropiación y circulación del valor, y también una renovada operación del Estado. La internacionalización en el ámbito de la relación capital-trabajo — es decir, la internacionalización de la "ley del valor" (Marini, 1996) — y en las relaciones sexo-género atravesaron cada vez más a los Estados nacionales, y estos expresan la relación social del capital y de las relaciones patriarcales. Efectivamente, es importante entender que el Estado y sus políticas no operan como agentes "neutrales" en términos de clase y género y raza/etnia. Por el contrario, las instituciones públicas son esenciales en la reproducción del orden establecido (Anzorena, 2013a). Así, aun si la crisis neoliberal en la región produjo cambios sustantivos en las modalidades de producción y producción social, las formas emergentes del neodesarrollismo permanecieron atravesadas por las relaciones sociales dominantes: capitalistas-patriarcales-racistas.

A través del proyecto neoliberal, en ALyc —en particular en Argentinalas fracciones dominantes del capital apuntalaron un proyecto de neodesarrollo hacia finales de los años noventa, el cual pudo consolidarse como superación dialéctica a la crisis del neoliberalismo (en Argentina, entre 1998 y 2002), y construyendo sobre su herencia una emergente valorización del valor. Este proyecto hegemónico fue producto del proceso histórico de las luchas sociales por reconfigurar el capitalismo patriarcal en crisis. En Sudamérica surgieron tres grandes proyectos cambiantes y flexibles de rearticulación posneoliberal (Féliz, 2015a; Thwaites \& Castillo, 2008). Por un lado, Colombia, Perú y Chile consolidaron patrones de reproducción del orden social dando mayor continuidad al neoliberalismo. Por su parte, Venezuela, Bolivia y, en menor medida, Ecuador, acompañando a Cuba, donde los pueblos pudieron irrumpir con mayor fuerza política, conformaron el eje del buenvivir. Con una disrupción de diferentes niveles de radicalidad, esos proyectos abonaron la transformación de algunas matrices capitalistas de la economía, la sociedad y el Estado. Finalmente, Brasil, Argentina, Uruguay y Paraguay consolidaron estrategias de neodesarrollo capitalista como proyecto societal de industrialización periférica y redistribución del ingreso. Con sus contradicciones y cambios en integrantes, estos procesos nacionales se articularon durante la era posneoliberal en la región. En ese marco, Argentina fue el ejemplo clásico de la estrategia del neodesarrollo.

\section{El saqueo como fundamento del proyecto neodesarrollista}

El neodesarrollismo en Argentina se consolidó a partir de 2003 apoyado en un nuevo modelo de Estado que pretendía ser el medio para proyectar la herencia 
neoliberal canalizándola productivamente para el capital (Féliz, 2012b). En la primera fase del neodesarrollo, el Estado argentino fue débil (Bonnet \& Piva, 2013) y generó algún contrapunto a la movilización popular y a las demandas antisistémicas de una porción políticamente organizada de la población. En apariencia autónomo ("politizado"), el Estado débil está obligado a responder a las demandas de diferentes fracciones de clase; sobre todo es forzado a dar cuenta parcial y fragmentariamente de las demandas de las clases populares (Féliz, 2016). La debilidad del Estado expresa la fragilidad de ciertas relaciones sociales que aun siendo dominantes son cuestionadas, al menos temporalmente.

Nacido de la crisis del proyecto neoliberal, pero no de su fracaso (Féliz, 2012b), el Estado argentino tuvo que reintegrar a las fuerzas sociales del trabajo en un marco favorable para su valorización y apropiación capitalista. Esto supuso el reforzamiento de un nuevo patrón de producción de valor sostenido en la transnacionalización generalizada del capital. Construida a través de la era neoliberal, se conformó una nueva "sociedad del capital" (Negri, 1992). Esta se consolidó a partir de renovados tipos de saqueo y acumulación originaria: el extractivismo como despojo de las riquezas naturales y los bienes comunes, la superexplotación de la fuerza de trabajo como expresión del saqueo de los cuerpos en el espacio del trabajo productivo y la multiplicación de los patrones de saqueo y apropiación violenta del cuerpo de las mujeres trabajadoras y pobres, como fundamento de modalidades que no se conocían del trabajo reproductivo. Este proceso se encuentra articulado a escala global y se pone de manifiesto exponencialmente en la configuración de enclaves geográficos específicos en nuestro territorio. No casualmente en ellos coinciden el extractivismo minero, petrolero o sojero, con la trata de personas y variopintos modos de militarización estatal, paraestatal o informalizada, es decir, la rapińa en sus diferentes expresiones (Segato, 2013).

En consecuencia, el neodesarrollismo se convierte en estrategia de desarrollo que refleja las necesidades de valorización de las fracciones hegemónicas dentro de las clases dominantes del capital: el gran capital transnacionalizado (Féliz, 2012a). Este proyecto buscaba exprimir cada "átomo" de riqueza social para consolidar un espacio de valorización competitivo a escala global, e inserto lo más perfectamente posible en las cadenas internacionales de valor. En el caso de Argentina eso implicaba, por un lado, la multiplicación y expansión de las modalidades de saqueo de las riquezas naturales (Merlinsky, 2016) de tal manera que permitieran la producción y apropiación capitalista de renta extraordinaria (Féliz, 2014). Por otra parte, el neodesarrollo suponía la consolidación de modalidades de uso de la fuerza de trabajo que permitieran superexplotarla para crear otros tantos modos de la ganancia extraordinaria (Féliz, 2014; Jaccoud et al., 2015). El reforzamiento del extractivismo permite estabilizar a Argentina 
como territorio para la producción de capital constante circulante; esencialmente, como materias primas de exportación de distintos niveles de manufactura: granos, harina, aceite y combustible elaborados con soja. Se cimentó así un patrón de inserción internacional dependiente para toda la producción de valores en el territorio argentino. En paralelo, el proyecto hegemónico intentó reformular la relación con la fuerza de trabajo, proceso que se convirtió en una normalización conflictiva (Dinerstein, Contartese \& Deledicque, 2008) del conflicto sociolaboral y la gestión productiva de esta mano de obra como capital variable al interior de una renovada composición del capital.

La caracterización general del nuevo proyecto hegemónico concluye en este punto (Féliz, 2012a). Sin embargo, un elemento fundamental, pero en general olvidado, es que las transformaciones del proyecto neoliberal configuraron otras modalidades de articulación entre el trabajo productivo y el reproductivo, reactualizando así el saqueo de los cuerpos de las mujeres y su explotación capitalista y patriarcal.

\section{Construcción hegemónica, género y políticas estatales}

La consolidación de un proyecto hegemónico neodesarrollista suponía entonces construir las condiciones para consolidar un patrón de valorización del capital apoyado en el saqueo de las riquezas naturales y la fuerza de trabajo, e implicaba normalizar conflictivamente la lucha social, en especial de los sectores más movilizados del pueblo trabajador.

La crisis neoliberal tuvo en su base una original recomposición política de la clase trabajadora (Féliz, 2011). La composición de clase refiere a la estructura de poder de clase que existe en la división del trabajo productivo y reproductivo, asociada a una particular organización del capital constante y variable entre trabajo remunerado y no remunerado, entre capital productivo y financiero, etc. (Cleaver, 1992). Mientras que con el neoliberalismo el capital buscaba estructurar una determinada composición de clase —es decir, una distribución del poder interclases/géneros e intraclase/géneros- que le permitiera controlar adecuadamente a la clase trabajadora para garantizar la acumulación, los trabajadores y trabajadoras en el conjunto de la fábrica social, sistemáticamente (aunque no necesariamente de modo consciente) enfrentaron, rechazaron y resistieron ese control. Buscaron así "recomponer" sus estructuras, formas organizativas y la distribución del poder, a fin de cambiar a su favor la correlación de fuerzas frente al capital (Cleaver, 1992).

A la salida del neoliberalismo, la composición política del pueblo trabajador involucraba al menos dos grandes fracciones dinámicas. Por un lado, una 
de dimensiones variables y trayectorias diversas que había nacido de las trabajadoras y trabajadores desocupados y expulsados a los márgenes del sistema por el impacto de la reestructuración neoliberal (Stratta \& Barrera, 2009). Por medio de un ciclo de protesta iniciado en los noventa, las organizaciones piqueteras desafiaron la configuración del proyecto societal conducido por el capital transnacionalizado. Estas fracciones nacieron a la lucha social por fuera de instituciones creadas a lo largo de casi un siglo de conformación de un Estado de bienestar periférico en Argentina. Por ello, sus demandas y disputas eran un reto mayúsculo para el Estado posneoliberal en construcción.

La otra fuerza social disonante a la caída del proyecto neoliberal la encarnaba un movimiento obrero organizado y una generación de activistas sindicales de base nacidos al calor del enfrentamiento contra el ajuste estructural. Sus demandas de recuperación salarial y una práctica de acción directa cuestionaban y presionaban a las cúpulas tradicionales de los sindicatos, las que debieron buscar cómo canalizar esas exigencias dentro del marco de la institucionalidad vigente (Schneider, 2013).

En tal sentido, un Estado débil pretendió integrar a los sectores con mayor potencial disruptivo, una tarea que fue parte de la construcción de la hegemonía de una fuerza política que, luego de la crisis de diciembre de 2001, se encaramó en el aparato estatal: el kirchnerismo, expresión de una fracción del movimiento peronista en el Partido Justicialista (Féliz, 2016).

Por una parte, las fracciones más formalizadas y organizadas de la fuerza de trabajo. Por otra, los sectores informalizados y/o desempleados en parte organizados en torno al movimiento piquetero. Las políticas sociales y laborales que se construyeron desde 2002 acompañaron la consolidación del proyecto de neodesarrollo y le fueron consustanciales. Se recuperaron para ello las viejas instituciones de la legislación laboral argentina para retomar algunas de las demandas de integración del movimiento obrero. En efecto, la revitalización de la negociación colectiva paritaria en el marco de los convenios colectivos de trabajo canalizó y contuvo las exigencias de los trabajadores más formalizados y masculinizados. La recuperación del crecimiento económico en los primeros años de la era que llegaba impulsó la caída en la tasa de desocupación y los niveles de empleo asalariado y por cuenta propia. El resultado fue que, al menos hasta 2008, crecieron las remuneraciones reales de los trabajadores varones - mayoritarios en el sector formal del mercado de empleo-, mientras que las mujeres —insertas sobre todo en los fragmentos informalizados y más precarizados - vieron que sus remuneraciones se estancaban o reducían, en el sector público, por ejemplo, reproduciendo la discriminación laboral en términos de ingresos.

El mercado laboral aparece segmentado, pero persiste un sesgo de género, racial e incluso etario. La precarización laboral, en tal sentido, no es homogénea. 
Las modalidades de trabajo "en negro"' (no registrado) incidieron con más fuerza sobre las mujeres trabajadoras, sobre todo entre las jóvenes y migrantes (del interior del país y de países vecinos). Este cuadro es inescindible de la desvalorización económica del trabajo reproductivo, que persistentemente supone una sobrecarga para las mujeres: la recuperación del empleo remunerado se superpone entre las mujeres con altas cargas de trabajo de reproducción y de cuidados, no pagado, en los hogares y comunidades. ${ }^{1}$ La rigidez de esta modalidad laboral no remunerada frena el ingreso pleno de la mayoría de las mujeres en el mercado de trabajo, lo que reduce en mucho su posibilidad de buscar y conseguir empleos a tiempo completo. Enfrentan, en consecuencia, un mercado de empleo peor pagado y más precarizado: menos horas de trabajo y menos pago por hora. Por otra parte, las mujeres encuentran en el mercado de trabajo una oferta de empleo sesgada a "empleos femeninos" que precarizan el trabajo: vinculados al cuidado o tareas domésticas, tienen su ejemplo paradigmático en el servicio doméstico. Este representa, según el INDEC, cerca de un quinto de las mujeres asalariadas y se encuentra fuertemente precarizado, ya que $77 \%$ de estas trabajadoras no están registradas en la seguridad social. Asimismo, el trabajo doméstico asalariado es una actividad muy racializada: las migrantes son el $25 \%$ del total de las trabajadoras de cuidados, situación que se suma a la informalidad laboral y la vulnerabilidad ante la discriminación y maltrato de empleadores y empleadoras (Paz \& Schteingart, 2011). Estos empleos socialmente desvalorizados, informales y mal pagados con frecuencia son la única opción para las mujeres migrantes, lo que manifiesta la jerarquización y segmentación étnico-nacional y de género del mercado de trabajo (Magliano, 2015), y la extensión de modos de precarización laboral a escala transnacional.

Por otra parte, el Estado es uno de los empleadores que utiliza mano de obra precarizada en tareas de obra pública, mantenimiento y servicios de limpieza, seguridad, etc.: en 2014, un 23.1\% del empleo en la administración pública nacional era precario, esto es, trabajadores con contratos temporales o como parte de un extendido grupo de empleados que fungen como autónomos, pero en actividades permanentes del Estado y como único o principal empleado (IDEP-CTA, 2015). Incluso las políticas sociales con las que se normalizó la

1 Como fue señalado, el trabajo reproductivo cumple un papel esencial en la producción y reproducción material de la fuerza de trabajo y por lo tanto en la constitución social del capital variable. En su mayor parte, sobre todo cuando remite a las actividades de cuidado, ha recaído históricamente en las mujeres, quienes han sido forzadas a tomarlo en un régimen no remunerado. En 2013, según datos del INDEC, las mujeres argentinas realizaban casi el doble de horas de trabajo no remunerado que los varones: tenían 3.9 horas diarias de trabajo en "quehaceres domésticos" contra las 2.4 horas en el caso de los varones, y 6 horas en "cuidado de nińos, nińas y personas mayores" versus solo 3.8 horas de los varones (Rodríguez, 2015). 
disrupción de los piqueteros y otros movimientos territoriales no institucionalizados (Dinerstein, Contartese \& Deledicque, 2008) constituyen, en los hechos, precarización laboral que, en su mayor parte, ocupa mujeres de sectores populares. Pueden asimismo reforzar lazos clientelares institucionalizando, en muchos casos, la asignación de roles y tareas de cuidados a las mujeres. El uso de la cooperativa para la organización de muchos de estos programas institucionaliza el fraude laboral por parte del Estado. Y estas mismas políticas han mantenido la evolución en el valor monetario de los beneficios de las diferentes modalidades de cooperativas de trabajo, subsidios para trabajo comunitario y programas de transferencia condicionada, las cuales están muy por debajo de la evolución general de las remuneraciones de los asalariados formalizados.

\section{¿Nuevas políticas sociales, viejos roles de género?}

De la era neodesarrollista argentina puede señalarse el reconocimiento de algunos derechos ciudadanos mediante una normativa que considera las disidencias sexo-genéricas: Ley de Matrimonio Igualitario aprobada en 2010, Identidad de género aprobada en 2012, Ley de Protección Integral a las Mujeres aprobada en 2009, Ley 26.364 de Prevención y Sanción de la Trata de Personas y Asistencia a sus Víctimas, sancionada en 2008, y la Ley de Salud Sexual y Procreación Responsable de 2003. Aun así, la histórica demanda de legalización del aborto permanece negada (CNDALSG, 2013). Y aunque esta legislación tiene serias dificultades para implementarse, supuso un reconocimiento de derechos relacionados con las desigualdades genéricas.

Estas iniciativas coexistieron durante todo el kirchnerismo de forma tensa y contradictoria con lo que Fraser (1997) denomina "políticas sociales de redistribución" para las mujeres pobres y trabajadoras. Se produjo así una ambivalencia estatal (Anzorena, 2013a), pues detrás de una retórica de ampliación de derechos para las mujeres en tanto ciudadanas, hay una negativa a reconocer problemáticas de género relativas a determinaciones de clase social y raza. Y persiste la ausencia de políticas laborales y sociales que distribuyan la riqueza social a las mujeres pobres y racializadas. Esta ambivalencia muestra que no se han cuestionado la división social y sexual del trabajo, la feminización de la pobreza y los mecanismos de segregación racial que reproducen la estructura social.

Desde el punto de vista de la articulación del trabajo productivo y reproductivo, las viejas y nuevas políticas replicaron los roles tradicionalmente asignados a mujeres y varones. Las políticas laborales del Ministerio de Trabajo se dirigen esencialmente a los varones, que son mayoría en el empleo formal. Mientras que las políticas sociales surgidas del Ministerio de Desarrollo Social, en los hechos 
se orientan a las mujeres que son la mayoría de la población precarizada. Este sesgo muestra que detrás de ellas permanece un estereotipado modelo de familia nuclear y heterosexual desde el que se conciben las políticas sociales de redistribución (Anzorena, 2013b). En específico, las políticas sociales dieron pauta a una nueva "economía de los cuidados" (Rodríguez Enríquez, 2005): las actividades de "cuidado" que incluyen la producción de los valores de uso necesarios para la reproducción cotidiana de las personas son el eje articulador de esas políticas y las mujeres son sus principales beneficiarias.

Las políticas sociales han estado históricamente dirigidas a la producción de los valores del cuidado como destino natural y asentado en el esfuerzo de las mujeres. En su origen, en 2002, el Plan Jefes y Jefas de Hogar Desocupados (PJJHD) se construyó como un masivo programa de transferencia condicionada de ingresos (TCI) que, con el apoyo de los organismos internacionales de crédito como el BID o el Banco Mundial, conformó un nuevo paradigma de políticas sociales de aplicación universal, pero de beneficios básicos (Molina, 2006). ${ }^{2}$

Estas nuevas políticas presentaron una retórica fundada en el supuesto cambio de paradigma en cuanto a la protección social y los derechos ciudadanos, con soluciones que buscarían superar los problemas de políticas previas. Basadas en la idea de que las personas actúan como agentes económicos racionales que aspiran a optimizar su situación, estas políticas refuerzan el precepto que asigna a las personas pobres la responsabilidad individual de su situación de pobreza y en sus acciones (agencia) la posibilidad de superarla (Rodríguez Enríquez, 2011). En la práctica, esas políticas se utilizan para paliar las consecuencias sociales de las políticas neoliberales y sus crisis, enfocándose en los sectores más empobrecidos, y son parte de la estrategia de contención del conflicto social que en Argentina combinó mecanismos de represión y judicialización de la protesta y la pobreza (Féliz \& Pérez, 2007; Anzorena, 2013b).

El mencionado PJJHD significó una transferencia de ingresos que reconoció el lugar central de las mujeres como articuladoras de las redes barriales y los movimientos territoriales. Su reemplazo en 2003, combinando el Plan Familias para los considerados no empleables y el Seguro de Capacitación y Empleo (SCE) para los potencialmente empleables (Pérez \& Brown, 2015), comienza a operar un desplazamiento cargado de un sesgo de género que ratificará a las

2 Estas políticas sociales presentan rasgos comunes con las destinadas a los sectores llamados informales (aunque son sobre todo no asalariados), que los Estados promueven con el auspicio de los organismos multilaterales de crédito en el continente. Poseen tres principales características: $a$ ) ser transferencias monetarias, $b$ ) estar orientadas a poblaciones específicas (focalización) y $c$ ) exigir requisitos a los beneficiarios (condicionalidad) (Fiszbein \& Schady, 2009). En la actualidad, existen en América Latina alrededor de 1240 programas de transferencia condicionada, que operan en 18 países de la región y cubren a más de 25 millones de hogares y cerca de 113 millones de personas (19\% de la población de la región). 
mujeres como cuidadoras con la responsabilidad de garantizar la protección de las niñas y los niños en cuanto a la asistencia a controles sanitarios y el cumplimiento de la escolaridad (Anzorena, 2013b). La creación de la Asignación Universal por Hijo (AUH) en 2009 consolidó las políticas que ratifican a las mujeres en un rol de cuidadoras. Este programa da un beneficio monetario por cada hijo o hija a las familias sin adultos ocupados o con personas en empleos precarios —es decir, sin seguridad social— con el único requisito de garantizar los controles sanitarios y educativos citados: el $94.4 \%$ de quienes cobran la AUH son mujeres (ANSES, 2012).

Dichos programas se sustentan en una perspectiva que promueve el paso de la idea de condicionalidad a la de corresponsabilidad entre el Estado y quienes perciben el ingreso. Con una nueva retórica política que subraya el regreso del Estado, ahora con políticas sociales masivas, las mujeres son forzadas a cargar con la responsabilidad de superar su situación de pobreza que, por otra parte, se origina en la simbiosis de capitalismo y patriarcado. Y son puestas a enfrentar las consecuencias negativas de la crisis general del régimen del capital con los pocos medios materiales que se les da a través de los programas de transferencias de ingreso.

Asimismo, estas políticas —a medio camino entre el empleo y el desempleo, entre la inclusión y la exclusión social— no están destinadas a las mujeres en tanto sujeto oprimido en las relaciones sociales de géneros y sexuales, sino en tanto responsables, cuidadoras de los nińos y niñas vulnerables. Al imponer requisitos para el cobro relacionado con la salud y la escolaridad de los hijos e hijas, se corre el eje a la educación, capacitación y reinserción laboral de las mujeres; es decir, aquello que podría mejorar su "empleabilidad". De esa manera, en contradicción con las políticas de reconocimiento, como las denomina Fraser (1997), las nuevas políticas sociales (marginalmente redistributivas) están reforzando la asignación de las tareas domésticas y de cuidados en las mujeres-madre, reproduciendo así la división sexual del trabajo que es un pilar de la sociedad patriarcal, racista y capitalista. La AUH se dota de legitimidad social porque se vincula con la función social asumida como femenina de la maternidad, y porque así se refuerza la complementariedad heterosexual en la familia y, más en general, en la división sexual del trabajo: mientras que el empleo se asocia a lo masculino, el trabajo doméstico, de cuidado y comunitario se ratifica como femenino (Anzorena, 2013b). La familia nuclear y heterosexual se mantiene como el modelo estereotipado a partir del cual se elaboran las políticas sociales de redistribución.

Reafirmando el carácter patriarcal de las políticas sociales - y por tanto, del Estado_- en 2013 se lanza la política Ellas Hacen (EH) que se focaliza en las mujeres de sectores populares como parte del Programa Ingreso Social más Trabajo (PIST, "Argentina Trabaja"). Consiste en un incentivo económico des- 
tinado a algunas mujeres que reciben la $\mathrm{AUH}$, pero condicionado a realizar trabajo comunitario, cumplir capacitaciones y presentar terminalidad educativa. Según fuentes oficiales, el plan estaba destinado a cien mil mujeres (Ministerio de Desarrollo Social, 2015) que deberían acreditar su pertenencia a grupos de vulnerabilidad extrema: mujeres víctimas de violencia machista, madres solas o solteras y con tres o más hijos o hijas a su cargo. Más allá de los discursos del Ministerio de Desarrollo Social sobre el "empoderamiento" de las mujeres y la igualdad de género que impulsa el programa (Ministerio de Desarrollo Social, 2015), no se trata de una política universal para las mujeres desempleadas de todo el país en tanto desfavorecidas en las relaciones de poder sociales, por el contrario, se enfoca a mujeres (de algunas provincias del país) en tanto madres, y excluye a las que no tienen hijos o hijas o que los tienen pero ya son mayores. De esta manera, el EH otorga preeminencia a los derechos de los hijos e hijas por encima de los derechos de las mujeres, pero al mismo tiempo no considera la sobrecarga de las mujeres por el trabajo reproductivo que realizan ni impulsa estrategias públicas para otra distribución. Igualmente, el peso de los beneficios recibidos en relación con la autonomía económica de las mujeres es ambiguo, porque se trata de un ingreso percibido a condición de acreditar la atención de la situación de los hijos e hijas e incluso, en algunos casos, deslinda a los varones de aportar a la manutención de los hijos (Molyneux, 2003; Rodríguez Enríquez, 2011; Anzorena, 2013a).

Podemos sugerir que este conjunto de políticas sociales, incompatibles con otras actividades registradas para las mujeres, fomentan la permanencia en el hogar de las beneficiarias y en las tareas asociadas a su cuidado. Son políticas que no proveen medios económicos suficientes ni promueven el empoderamiento de las mujeres. Por el contrario, a partir del reconocimiento monetario insuficiente de los trabajos de cuidados, impulsan soluciones de bajo costo para el cuidado familiar y el trabajo comunitario y desincentivan la participación femenina en el mercado de trabajo formal. De este modo, tales programas aprovechan los roles de género tradicionales y el trabajo no remunerado de las mujeres, sin crear condiciones para que los varones se involucren en las tareas de cuidados y sin proponer mecanismos y dispositivos estatales para resolver el reparto desigual de esas cargas. Una reflexión aparte merecería el desarrollo de estrategias individuales y colectivas por parte de las mujeres y las organizaciones populares para colectivizar, hacer comunitario y cuestionar la distribución de dichos trabajos para así otorgar significados alternativos a estos ingresos y suscitar prácticas emancipadoras. ${ }^{3}$

3 La literatura sobre los movimientos piqueteros ha destacado la resignificación que estas organizaciones realizan de los subsidios de desempleo asignados por el Estado (Svampa \& Pereyra, 2003: p. 221). Pero, 
Dichas políticas sociales, orientadas en efecto a las mujeres en tanto cuidadoras o madres, han frenado su ingreso al mercado laboral. Luego de varias décadas de aumento en la tasa de actividad de las mujeres en ese mercado, en los años dos mil este índice se estanca durante el primer lustro neodesarrollista. ${ }^{4} \mathrm{Y}$ para las mujeres de los sectores populares se observa una caída en su participación para el último lustro. ${ }^{5}$ Cabe decir que se mantiene la prevalencia del empleo femenino en las actividades de servicios (Falquet, 2016a) vinculadas al Estado (salud y educación), en el comercio y en casas como servicio doméstico. ${ }^{6}$

\section{Neodesarrollo y patriarcado}

El neodesarrollo en Argentina se fundó entonces en tres pilares. Por un lado, el saqueo de las riquezas naturales, la superexplotación del trabajo y nuevas modalidades de apropiación del trabajo reproductivo a cargo en gran medida de las mujeres. En segundo lugar, se apoya en un paquete de políticas laborales que canalizan las demandas de las fracciones trabajadoras más organizadas (en particular, los varones) y en la articulación de políticas sociales focalizadas con otras de enfoque más universal que integran parcial y conflictivamente a las fracciones sociales más excluidas (en especial, las mujeres). Y, en tercer lugar, un amplio abanico de políticas de reconocimiento (Fraser, 1997) de derechos ciudadanos que, si muy limitado, permitió consolidar la hegemonía de un proyecto societal acuñado como de crecimiento con inclusión social (Arroyo, 2004).

La consolidación del proyecto neodesarrollista se produjo en tanto el mito de la inclusión apareció como posible en un contexto expansivo, lo que sucedió hasta 2008. Sin embargo, esa consolidación no operó sin contradicciones (Féliz, 2015b). En referencia a las articulaciones entre neodesarrollismo, extractivismo y patriarcado, esas contradicciones se expresaron de manera específica.

fundamentalmente señalan que las mujeres, con su presencia mayoritaria y cotidiana en organizaciones populares, crean novedosas prácticas y sentidos vinculados a la autogestión. Esto se produce mediante mecanismos de colectivización del trabajo, utilizando como base del ingreso las políticas sociales que originalmente fueron planteadas como de beneficio individual (Díaz, 2015).

4 Según datos del CEDLAs (con información del INDEC), luego de aumentar cinco puntos porcentuales entre 1998 y 2002, la tasa de actividad para las mujeres adultas —-25-64 ańos de edad—se estancó entre 2003 y 2013 en torno al $69.3 \%$, lo que contrasta con el $92.7 \%$ promedio para los varones.

5 Entre 1998 y 2002 la tasa de actividad sube casi once puntos porcentuales entre las mujeres del primer quintil de ingresos (las más pobres); entre 2003 y 2013, por el contrario, cae fuertemente de 46.8 a $35.9 \%$.

6 Según el CeDLAs (con datos del INDEC) entre 2003 y 2013 (en promedio) el 30.3\% de las mujeres trabajaban con remuneración en áreas de educación y salud (solo $11.7 \%$ de los varones), el 21.9\% en comercio ( $24.4 \%$ de los varones) y $17.8 \%$ en servicio doméstico (solo $0.5 \%$ de los varones). 
La expansión capitalista en Argentina se sostiene en la superexplotación de la fuerza de trabajo, aunque ello presenta sesgos de género y clasistas. Mientras que hacia la mitad de la primera década del dos mil se robustece un patrón de inclusión parcial y precario de la fuerza de trabajo formal masculinizada, entre las mujeres se dan mayores patrones de exclusión y superexplotación. Esto ocurre aun cuando las políticas de asistencia social —ahora masivas pero básicas- motivan que las mujeres ya no busquen activamente un empleo, y aun si esas políticas no las retiran definitivamente del mercado de trabajo; las mujeres persisten como parte de una fuerza laboral latente dispuesta a tomar un empleo si surge la oportunidad (Marx, 1995). Por otra parte, la superexplotación laboral se concentra en las mujeres, las cuales toman los empleos peor pagados y más precarizados, como los servicios personales — sobre todo el doméstico, pero se podría agregar el "trabajo sexual"-, el comercio y el empleo público. La superexplotación capitalista está cargada de patriarcado.

Las políticas sociales que en realidad colocan a las mujeres en el tradicional papel de cuidadora o madre operan contradictoriamente, dado que les reconocen ciertos derechos ciudadanos en la etapa constitutiva del neodesarrollo. Es una contradicción que expresa en parte el reconocimiento social de la lucha histórica del movimiento de mujeres. En efecto, las políticas estatales, tanto redistributivas como de reconocimiento, registran discontinua y fragmentariamente las luchas diversas de las mujeres y reconocen su poder social. Si bien en ambos casos sirven de mecanismos para canalizar políticamente las exigencias de igualdad social, lo hacen de modo de normalizarlas conflictivamente, intentando la neutralización de su potencia disruptiva. Por eso buscan construir patrones de organización familiar que reproduzcan la centralidad de las mujeres en el trabajo de cuidados y reproducción de la vida, y enfatizan la maternidad como práctica obligatoria de las mujeres, sobre todo para las heterosexuales. Es una centralidad clave para garantizar la reproducción de la fuerza de trabajo y la vida que permitan que la "familia" (y esencialmente las mujeres) siga siendo el dispositivo de absorción de los ajustes del capitalismo patriarcal (buffer) en tiempos de crisis. En el neodesarrollo, entonces, la organización social del trabajo de cuidado sigue cargando en las mujeres la obligación de contener y compensar los impactos del ajuste al interior del espacio productivo en el ciclo del capital. A diferencia de la etapa neoliberal, detrás de una impronta de avance estatal en el desarrollo de políticas sociales inclusivas, se construye una modalidad de articulación del patriarcado con el capital y en el capital. Ahora, con la transferencia condicionada de ingresos, se intenta sujetar de nuevo a las mujeres en relaciones familiares normalizadas dentro del patriarcado moderno.

Por otro lado, la lucha de las mujeres y su impacto en el Estado entra en contradicción abierta con el extractivismo como ruta particular del saqueo. En 
efecto, las modalidades más recientes del extractivismo en la era del neodesarrollo multiplican las de la violencia contra las mujeres. La naturaleza violentada tiene su reflejo en la violencia contra las mujeres, de forma en particular virulenta contra aquellas que deciden resistir el acoso y la opresión. La resistencia femenina exacerba la violencia machista. En los enclaves extractivistas, vinculados sobre todo al saqueo de recursos de hidrocarburos y mineros, esta dinámica de violencia se multiplica de modos diversos entre los que la prostitución es una de sus expresiones más brutales. Además, la expulsión de las mujeres pobres hacia las periferias precarizadas de las ciudades es producto de la especulación inmobiliaria y de la recirculación de la renta extraordinaria hacia los espacios urbanos.

La heterocirculación de las mujeres en la ruta del petróleo y en el desarrollo de las redes internacionales del cuidado amplían esas violencias (Falquet, 2016a, 2016b). A contramano de la lucha de las mujeres, el extractivismo exacerba los ataques hacia ellas y hacia los cuerpos feminizados. El reconocimiento de la "agencia femenina" enfrenta expresiones agigantadas del machismo: el asesinato y el abuso sintetizan la articulación criminal del patriarcado y el extractivismo. Si este último en la era del neodesarrollo supone construir "zonas de sacrificio" - donde las vidas, la naturaleza y las prácticas comunitarias devienen descartables-, el cuerpo-territorio de las mujeres se convierte en espacio colectivo de apropiación y destrucción (Segato, 2014; García, 2015). La multiplicación de las resistencias de las mujeres y la expansión de la violencia extractivista en el continente en las últimas décadas, tienen como correlato el incremento exponencial del femicidio y la trata de mujeres (Feministas de Abya Yala en Resistencia, 2016; Mansilla, 2017).

El neodesarrollismo es un proyecto de desarrollo del capital en la periferia que se constituye a partir de la articulación contradictoria de capitalismo, extractivismo y patriarcado. El saqueo como superexplotación del trabajo directamente productivo de valor se soporta inevitablemente en la superexplotación del trabajo reproductivo y de cuidados que replican el sustrato metaestable del patriarcado y se sostienen en él. Esas modalidades del saqueo de los cuerpos se conciertan perfectamente con la superexplotación consciente de la naturaleza y los bienes comunes, pero son impensables sin determinadas políticas estatales y la violencia sistémica. Efectivamente, la rapiña de cuerpos y territorios se compensa y asegura con políticas asistencialistas o represivas (Aráoz, 2013). De esa manera, las políticas sociales amortiguan las consecuencias de la superexplotación del trabajo en el espacio productivo, del deterioro en las políticas estatales de carácter universal y socialmente integrador — sobre todo salud y educación públicas-y de la desarticulación de los espacios de socialización colectiva en los barrios y ciudades. Detrás de esos amortiguadores están las mujeres —en especial, 
en las familias más pobres_ que se ven forzadas a absorber y canalizar a través de sus cuerpos las crecientes presiones de la crisis sistémica del neodesarrollo.

Este proceso es definido por Rita Segato como una modalidad donde "la depredación y la rapiña del ambiente y de la mano de obra se dan las manos con la violación sistemática” (Segato, 2013: p. 44) que involucra un avance del capital, materializado en Estados y corporaciones. Ello implica una destrucción de la naturaleza y del mundo sin precedentes que afecta a los bienes comunes y se convierte en una rapiña de los cuerpos. Así, como señala Segato, la violencia permite el ejercicio de una soberanía, de un control territorial, que se expresa en su capacidad de acción irrestricta sobre los cuerpos (Díaz, 2013).

Esta depredación del territorio y la fuerza de trabajo es sistemática y da lugar a fenómenos globales que gestan un escenario signado por la violencia y la destrucción: las maquilas como prototipo de la realidad laboral, la guerra territorial protagonizada por el narcotráfico, la difusión de los códigos carcelarios a barrios enteros, la desaparición de mujeres a manos de mafias, y la proliferación de sicarios que multiplican crímenes por encargo. En las últimas décadas, los movimientos ambientalistas, indígenas, feministas y sociales han denunciado esta articulación de extractivismos, rapiña de los cuerpos y violencia, como modalidades neocoloniales de explotación y saqueo, que hacen blanco principalmente en las mujeres pobres, racializadas y/o migrantes (Segato, 2014; Falquet, 2016a, 2016b; Cabnal, 2010). El asesinato y persecución de mujeres que encabezan luchas en defensa de los territorios y los cuerpos constatan estas resistencias encarnadas en el corazón de la vida comunitaria y popular.

\section{Conclusiones preliminares}

El capitalismo y el patriarcado son modalidades de producción de la sociedad que se articulan simbióticamente. Ambos cambian e involucran la acción del Estado en diferentes momentos del tiempo y de espacios geográfico-territoriales. En regiones periféricas a nivel global, como sucede en América Latina, el capitalismo patriarcal surgió aliado a dinámicas coloniales de racialización y opresión que siguen vigentes en la división del trabajo y en la explotación de los cuerpos.

En este texto se ha buscado mostrar cómo el proyecto societal neodesarrollista nacido en Argentina de la crisis del proyecto neoliberal a comienzos del siglo XXI, presenta alternativas novedosas para la conformación de un modo particular de ser del capitalismo patriarcal.

En este marco, las políticas estatales son contradictorias, dado que intentan canalizar los conflictos de clase dentro de las modalidades de la valorización 
ampliada del valor. Esas políticas involucran formas de apropiación de la naturaleza/bienes comunes y el trabajo en general, y también suponen estrategias únicas que proyectan la apropiación colectiva del trabajo de reproducción o de cuidados, realizado en su mayor parte por las mujeres y personas feminizadas. De las políticas sociales destinadas a las mujeres pobres, puede señalarse que no han cuestionado la división social y sexual del trabajo, la feminización de la pobreza y los mecanismos de segregación racial que reproducen la estructura social, lo que genera que sean estas mujeres las que continúen actuando como contención y regulación de los impactos de las políticas económicas.

En la década larga del neodesarrollo (2002-2015, al menos) esas estrategias de integración y expropiación del cuerpo y el trabajo de las mujeres pudieron actuar articuladamente con la producción general de riqueza material.

Aun así, del mismo modo en que los usos de la naturaleza y el trabajo masculino productivo se desarrollaron en contradicción, la apropiación general del tiempo y el trabajo de las mujeres encontró resistencias ligadas, por ejemplo, a las estrategias de organización colectiva y autogestión comunitaria. Estas formas de organización y enfrentamiento deben analizarse más profundamente para comprender mejor cómo se relacionan con las formas más tradicionales de la lucha social en el capitalismo. Queda pendiente ampliar la comprensión de cómo los cambios en la organización del trabajo reproductivo operan como mediación y contradicción frente al trabajo directamente productivo de valor y plusvalor.

\section{Referencias}

Amorós, C. (1985). Hacia una crítica de la razón patriarcal. Madrid: Anthropos.

Anzorena, C. (2013a). Mujeres en la trama del Estado. Una lectura feminista de las politicas públicas. Universidad Nacional de Cuyo: Ediunc.

Anzorena, C. (2013b). Desigualdades que persisten: el lugar de las mujeres en las políticas sociales (Argentina 2000 a 2010). Nómadas. Revista Crítica de Ciencias Sociales y Jurídicas, (Número especial: América Latina). Recuperado el 4 de mayo de 2018, de http://ri.conicet .gov.ar/admin/bitstream/11336/692/1/claudiaanzorena_NOMADAS.pdf

Aráoz, H. Machado (2013). Orden neocolonial, extractivismo y ecología política de las emociones. RBSE-Revista Brasileira de Sociologia da Emoção, 12(34), 11-43.

Arroyo, D. (2004). Ante el actual escenario latinoamericano. Hacia un nuevo modelo de desarrollo: crecimiento con inclusión social. Sociedade em Debate, 10(3), 135-147. 
Bonnet, A. \& Piva, A. (2013). Un análisis de los cambios en la forma de Estado en la posconvertibilidad. En Grigera, J. (Ed.). Argentina después de la convertibilidad (2002-2011) (pp. 3-32). Buenos Aires: Imago Mundi.

Cabnal, L. (2010). Acercamiento a la construcción de la propuesta de pensamiento epistémico de las mujeres indígenas feministas comunitarias de Abya Yala. En Acsur-Las Segovias (Coord.). Feminismos diversos: el feminismo comunitario (pp. 11-25). España: ACsur/Las Segovias. Recuperado el 4 de mayo de 2018, de https://porunavidavivible.files.wordpress .com/2012/09/feminismos-comunitario-lorena-cabnal.pdf

Cecchini, S. \& Madariaga, A. (2011). Programas de Transferencias Condicionadas. Balance de la experiencia en América Latina y el Caribe. Santiago: CEPAL/Asdi.

Cielo, C. \& Vega, C. (2015). Reproducción, mujeres y comunes. Leer a Silvia Federici desde el Ecuador actual. Nueva Sociedad, (256), marzo-abril, pp. 132-144.

Cleaver, H. (1992, 13 de enero). Theses on secular crisis in capitalism: The insurpassability of class antagonism. Rethinking Marxism Conference. Amherst, Massachussets: University of Massachusetts .

CNPALsg (Campaña Nacional por el Derecho al Aborte Legal, Seguro y Gratuito) (2013). A la Comisión de Reforma del Código Penal. Documento. Publicado el 20 de noviembre de 2013. Recuperado el 15 de agosto de 2016, de http://www.abortolegal.com.ar/documento -enviado-por-la-campana-a-la-comision-bicameral/

Dalla Costa, M. (2009). Dinero, perlas y flores en la reproducción feminista. Madrid: Akal.

Dalla Costa, M. \& Selma, J. (1972). El poder de la mujer y la subversión de la comunidad. México: Siglo XXI.

De Angelis, M. (2012). Marx y la acumulación primitiva. El carácter continuo de los "cerramientos" capitalistas. Theomai, (26). Recuperado el 4 de mayo de 2018, de http://www .revista-theomai.unq.edu.ar/

Díaz Lozano, J. A. (2013). Conferencia de Rita Segato "Género y derechos humanos: una articulación indispensable para profundizar la transformación social de nuestros pueblos”. Desgrabación del audio. 29 de julio de 2013. La Plata: Facultad de Periodismo y Comunicación Social (UNLP).

Díaz Lozano, J. (2015). Movimiento en Femenino. Trayectorias de vida de mujeres que participan en organizaciones populares. En Nateras, A., Medina, G. \& Sepúlveda, M. (Coords.). Escrituras emergentes de las juventudes Latinoamericanas (pp. 49-97). México: Gedisa. 
Falquet, J. (2016a). Hacia un análisis feminista y dialéctico de la globalización neoliberal. El peso del complejo militar-industrial sobre las mujeres globales. En Cátedra Libre Virginia Bolten (Comp.). Cuadernillo Boltereano. La Plata: Cátedra Libre Virginia Bolten.

Falquet, J. (2016b). De los asesinatos de ciudad Juárez al fenómeno de los feminicidios. Nuevas formas de violencia hacia las mujeres. En Cátedra Libre Virginia Bolten (Comp.). Cuadernillo Boltereano. La Plata: Cátedra Libre Virginia Bolten.

Federici, S. (2013). Revolución en punto cero. Trabajo doméstico, reproducción y luchas Feministas. Madrid: Traficantes de Sueńos.

Federici, S. (2011). Calibán y la bruja. Mujeres, cuerpo y acumulación originaria. Buenos Aires: Tinta Limón Ediciones.

Féliz, M. (2016). Till death do as apart? Kirchnerism, neodevelopmentalism and the struggle for hegemony in Argentina, 2003-2015. En Schmitt, I. (Comp.). The Three Worlds of Social Democracy: A Global View from the Heartlands to the Periphery (pp. 91-106). Londres: Pluto Press.

Féliz, M. (2015a). ¿Qué hacer... con el desarrollo? Neodesarrollismos, buenvivir y alternativas populares. Sociedad y Economía, (28), 29-49.

Féliz, M. (2015b). Limits and barriers of neodevelopmentalism: Lessons from Argentina's experience, 2003-2011. Review of Radical Political Economics, 47(1), 70-89.

Féliz, M. (2014). Neo-developmentalism, Accumulation by Dispossession and International Rent-Argentina, 2003-2013. International Critical Thought, 4(4), 499-509.

Féliz, M. (2012a). Sin clase. Neodesarrollismo y neoestructuralismo en Argentina (2002-2011). Século XXI: Revista de Ciências Sociais, 2(2), 9-43.

Féliz, M. (2012b). Neo-Developmentalism Beyond Neoliberalism? Capitalist Crisis and Argentina's Development since the 1990s. Historical Materialism, 20(2), 105-123.

Féliz, M. (2011). Un estudio sobre la crisis en un pais periférico. La economía argentina del crecimiento a la crisis, 1991-2002. Buenos Aires: El Colectivo.

Féliz, M. \& Pérez, P. E. (2007). ¿Tiempos de cambio? Contradicciones y conflictos en la política económica de la posconvertibilidad. En Boyer, R. \& Neffa, J. C. Salidas de crisis y estrategias alternativas de desarrollo. La experiencia argentina (pp. 319-352). Buenos Aires: Miño \& Dávila. 
Feministas del Abya Yala en Resistencia (2016). Manifiesto. Recuperado el 10 de agosto de 2016, de https://www.frentedariosantillan.org/feministas-del-abya-yala-en-resistencia/

Fiszbein, A. \& Schady, N. (2009). Transferencias monetarias condicionadas. Reduciendo la pobreza actual y futura. Washington, D. C.: Banco Mundial.

Fraser, N. (1997). ¿De la redistribución al reconocimiento? Dilemas en torno a la justicia en una época "postsocialista" . En Fraser, N. (Ed.). Iustitia Interrupta: Reflexiones críticas desde la posición "postsocialista" (pp. 17-54). Bogotá: Siglo del Hombres Editores.

García Torres, M. (2015). Apuntes para un diálogo entre economía ecológica y economía feminista. Ecología Política: Cuadernos de Debate Internacional, (50), pp. 39-44.

Gargallo, F. (2002). El feminismo múltiple: prácticas e ideas feministas en América Latina. En Femenías, M. L. (Comp.). Perfiles del feminismo iberoamericano. Volumen 1 (pp. 103-130). Buenos Aires: Catálogos.

Haraway, D. (2004). Testigo_Modesto@Segundo_Milenio.HombreHembra@_Conoce_Oncoratón . Barcelona: uoc.

Haraway, D. (1995). Conocimientos situados: la cuestión científica en el feminismo y el privilegio de la perspectiva parcial. En Haraway, D. (Ed.). Ciencia, cyborgs y mujeres. La invención de la naturaleza. (pp. 313-346). Madrid: Cátedra.

Hartmann, H. (1985 [1979]). El infeliz matrimonio entre marxismo y feminismo: hacia una unión más progresista. Teoría y Política, (12-13), 5-29.

Harvey, D. (2005). El nuevo imperialismo: acumulación por desposesión. Buenos Aires: CLACso.

IDEP-CTA. (2015). Apuntes sobre la precarización laboral en el sector público nacional. Documento de Trabajo, s/n. Buenos Aires: IDEP-CTA. Recuperado el 4 de mayo de 2018, de http://www .ateargentina.org.ar/idep/documentos/134Analisis_sobre_la_precarizacion_laboral_en_el_ sector_publico_nacional.pdf

Izquierdo, M. J. (1983). Las, los, les (lis, lus). El sistema sexolgénero y la mujer como sujeto de transformación social. Barcelona: LaSal.

Jaccoud, F., Arakaki, A., Monteforte, E., Pacífico, L., Grańa, J. \& Kennedy, D. (2015). Estructura productiva y reproducción de la fuerza de trabajo: la vigencia de los limitantes estructurales de la economía argentina. Cuadernos de Economía Crítica, (2), 79-112.

Lorde, A. (2003). La hermana, la extranjera. Artículos y conferencias. Madrid: Horas y Horas. 
Lugones, M. (2008). Colonialidad y Género. Tabula Rasa, (9), 73-101.

Mansilla, M. (2017, 10 de marzo). Migrantes del patriarcado. Página/12, Suplemento Las12. Recuperado el 12 de agosto de 2016, de https://www.pagina12.com.ar/24767-migrantesdel-patriarcado

Marini, R. M. (1996). Proceso y tendencias de la globalización capitalista. En Marini, R. M. \& Millán, M. (Coords.). La teoría social latinoamericana, Cuestiones contemporáneas, tomo IV (pp. 49-68). México: Ediciones El Caballito.

Marx, C. (1995). El capital, tomo 1, volumen 3. México: Siglo XXI.

Merlinsky, G. (Comp.). (2016). Cartografías del conflicto ambiental en Argentina 2. Buenos Aires: CLACSO/Fundación CICCUS.

Millett, K. (2010). Politica sexual. Madrid: Ediciones Cátedra.

Ministerio de Desarrollo Social. (2015). Ellas hacen. Mujeres en acción por una vida mejor. Recuperado el 18 de julio de 2016, de http://www.desarrollosocial.gob.ar/ellashacen

Molina, C. G. (Ed.). (2006). Universalismo básico. Una nueva politica social para América Latina. México: Planeta Mexicana.

Molyneux, M. (2003). Movimientos de mujeres en América Latina. Estudio teórico comparado. Valencia: Ediciones Cátedra.

Negri, A. (1992). Interpretation of the class situation today: Methodological aspects. En Bonefeld, W., Gunn, R. \& Psychopedis, K. (Eds.). Open marxism. Vol. II Theory and practice (pp. 69-105). Londres: Pluto Press.

Novick, M., Rojo, S. \& Castillo, V. (Comps.). (2008). El trabajo femenino en la posconvertibilidad. Argentina 2003-2007. Buenos Aires: CEPAL.

Paz, C. \& Schteingart, D. (2011). Mercado de trabajo y género. El caso de las empleadas domésticas. $10^{\circ}$ Congreso Nacional de Estudios del Trabajo. Buenos Aires: ASET.

Pérez Orozco, A. (2014). Subversión feminista de la economía. Madrid: Traficantes de Sueños.

Pérez, P. \& Brown, B. (2015). ¿¿Una nueva protección social para un nuevo desarrollismo? Políticas sociales en la Argentina posneoliberal. Estudios Sociales del Estado, (2), 94-117.

Rodríguez Enríquez, C. (2015). El trabajo de cuidado no remunerado en Argentina: un análisis desde la evidencia del Módulo de Trabajo no Remunerado. Serie Documentos de Trabajo: 
Políticas públicas y derecho al cuidado, núm. 2. Buenos Aires: Equipo Latinoamericano de Justicia y Género.

Rodríguez Enríquez, C. (2011). Programas de transferencias condicionadas de ingreso e igualdad de género: ¿Por dónde anda América Latina? Santiago de Chile: CEPAL.

Rodríguez Enríquez, C. (2005). Economía del cuidado y politica económica: una aproximación a sus interrelaciones. Trigésima octava reunión de la Mesa Directiva de la Conferencia Regional sobre la Mujer de América Latina. Mar del Plata: CEPAL.

Rubin, G. (1996). El tráfico de mujeres: Notas sobre la "economía política” del sexo. En Lamas, M. (Comp.). El género: la construcción cultural de la diferencia sexual (pp. 35-96). México: PUEG.

Sassen, S. (1991). The Global City. Princeton: Princeton University Press.

Schneider, A. (2013). Política laboral y protesta obrera durante la presidencia de Néstor Kirchner (2003-2007). En Grigera, J. (Comp.). Argentina después de la convertibilidad (2002-2011) (pp. 97-115). Buenos Aires: Imago Mundi.

Segato, R. L. (2014). Las nuevas formas de la guerra y el cuerpo de las mujeres. Puebla: Pez en el Árbol.

Segato, R. L. (2013). La escritura en el cuerpo de las mujeres asesinadas en Ciudad Juárez. Buenos Aires: Tinta Limón.

Segato, R. (2011). Género y colonialidad: en busca de las claves de lectura y de un vocabulario estratégico descolonial. En Bidaseca, K. \& Vazquez Laba, V. (Comps.). Feminismo y Poscolonialidad. Descolonizando el feminismo desde y en América Latina (pp.17-48). Buenos Aires: Ediciones Godot Argentina.

Stratta, F. \& Barrera, M. (2009). ¿ Movimientos sin clases o clases sin movimientos? Notas sobre la recepción de la teoría de los movimientos sociales en la Argentina. Conflicto Social, 2(1), 118-134.

Svampa, M. \& Pereyra, S. (2003). Entre la ruta y el barrio. La experiencia de las organizaciones piqueteras. Buenos Aires: Biblos.

Tabet, P. (2005). Las manos, los instrumentos, las armas. En Curiel, O. \& Falquet, J. (Comps.). El patriarcado al desnudo. Tres feministas materialistas: Colette Guillaumin-Paola Tabet-Nicole Claude Mathieu. Buenos Aires: Brecha Lésbica.

Thwaites Rey, M. \& Castillo, J. (2008). Desarrollo, dependencia y Estado en el debate latinoamericano. Araucaria. Revista Iberoamericana de Filosofía, Politica y Humanidades, 10(19), 24-45. 
Tristán, F. (1993). Feminismo y utopía. México: Fontamara.

Valdés Gutiérrez, G. (2001). Hacia un nuevo paradigma de articulación (no tramposo) de las demandas emancipatorias. Utopía y Praxis Latinoamericana, 6(14), 48-57.

Vivero Vigoya, M. (2016). La interseccionalidad: una aproximación situada a la dominación. Debate Feminista, (52), 1-17. 